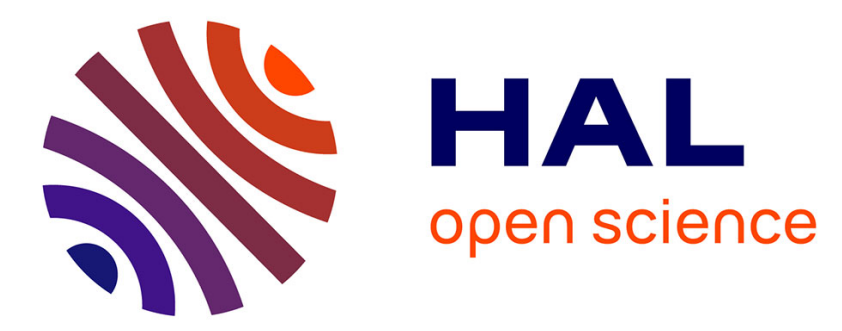

\title{
Les régimes dérogatoires en droit international des droits et libertés : approche comparée (Comité, Cour européenne et Cour interaméricaine des droits de l'homme)
}

\author{
Marie Rota
}

\section{To cite this version:}

Marie Rota. Les régimes dérogatoires en droit international des droits et libertés : approche comparée (Comité, Cour européenne et Cour interaméricaine des droits de l'homme). Cahiers de la recherche sur les droits fondamentaux , 2019, 10.4000/crdf.3850 . hal-03007479

\author{
HAL Id: hal-03007479 \\ https://hal.science/hal-03007479
}

Submitted on 24 Nov 2020

HAL is a multi-disciplinary open access archive for the deposit and dissemination of scientific research documents, whether they are published or not. The documents may come from teaching and research institutions in France or abroad, or from public or private research centers.
L'archive ouverte pluridisciplinaire HAL, est destinée au dépôt et à la diffusion de documents scientifiques de niveau recherche, publiés ou non, émanant des établissements d'enseignement et de recherche français ou étrangers, des laboratoires publics ou privés. 


\title{
Les régimes dérogatoires en droit international des droits et libertés: approche comparée (Comité, Cour européenne et Cour interaméricaine des droits de l'homme)
}

\author{
Marie ROTA \\ Maîtresse de conférences en droit public à l'université de Lorraine \\ Institut de recherches sur l'évolution de la nation et de l'État (IRENEE, EA 7303)
}

I. La création de clauses dérogatoires ou la consécration de l'approche dualiste des pouvoirs de crise

A. La consécration du modèle dualiste par le Pacte international relatif aux droits civils et politiques

$B$. La reprise du modèle international dans les conventions régionales

II. Un encadrement variable en fonction de la pratique des organes de contrôle

A. L'extension des normes indérogeables par le Comité des droits de l'homme et la Cour interaméricaine

B. L'exercice d'un contrôle plus poussé par le Comité et la Cour interaméricaine sur la mise en œuvre de la clause dérogatoire

C. Le retour à l'approche moniste en dehors du déclenchement de la clause dérogatoire: une tendance propre au juge européen

C'est par ces termes que Thorbjørn Jagland conclut sa lettre adressée à François Hollande le 22 janvier 2016. Ils permettent de saisir les risques de déviance de l'état
Le Conseil de l'Europe est prêt à apporter son assistance, si vous le jugez utile, pour que les réformes que vous avez annoncées s'inscrivent dans le respect des normes européennes relatives aux droits de l'homme. Je rappelle à cet égard qu'une fois l'état d'urgence levé, la Convention européenne des droits de l'homme s'appliquera sans dérogations aucune aux réformes qui seront alors en vigueur'.

1. T. Jagland, secrétaire général du Conseil de l'Europe, lettre au président de la République française, François Hollande, 22 janvier 2016, en ligne: http://rm.coe.int/CoERMPublicCommonSearchServices/DisplayDCTMContent?documentId=090000168059375b.

2. Olivier Cahn considère à ce titre que «le législateur français a, depuis la loi de 1996, progressivement institué un droit pénal de l'ennemi pour combattre le terrorisme - au point d’être parvenu à créer un dispositif répressif dérogatoire permanent» (O. Cahn, «"Cet ennemi intérieur, nous devons le combattre". Le dispositif antiterroriste français, une manifestation du droit pénal de l'ennemi», Archives de politique criminelle, nº 38, 2016, p. 95). 
que semble rappeler le secrétaire général du Conseil de l'Europe au regard des «réformes» visées ${ }^{3}$. En signalant à la France le caractère temporaire des dérogations à la Convention de sauvegarde des droits de l'homme et des libertés fondamentales ${ }^{4}$ admises au titre de son article $15^{5}$, il rappelle aussi implicitement l'existence d'un contrôle international en la matière, qui apparaît plus que jamais nécessaire ${ }^{6}$.

Qu'en est-il néanmoins d'un tel contrôle? Notons dès à présent que, sur la scène internationale, seuls les mécanismes pouvant faire l'objet d'une saisine par les individus apparaissent comme réellement efficaces lorsqu'il s'agit de protéger les droits et libertés. De ce point de vue, on a pu assister à leur multiplication comme en atteste la création de droits de recours individuel en vue de protéger plusieurs conventions internationales relatives aux droits de l'homme'.

Or, la plupart d'entre elles consacrent des clauses dérogatoires ${ }^{8}$ qui sont construites sur un même modèle. Les articles 4 du Pacte international relatif aux droits civils et politiques 9,27 de la Convention américaine relative aux droits de l'homme ${ }^{10}, 15$ de la Convention européenne, $\mathrm{F}$ de la Charte sociale européenne révisée et 4 de la Charte arabe des droits de l'homme, pour ne citer que ceux-là, se décomposent en plusieurs paragraphes similaires. Le premier autorise de déroger aux droits dans des circonstances exceptionnelles; le deuxième - lorsqu'il existe prévoit l'existence de droits indérogeables, y compris dans de telles circonstances; le dernier pose une obligation de notification à la charge de l'État concerné qui doit informer l'ensemble des États parties aux conventions de son recours aux clauses dérogatoires.

C'est pourquoi une "approche comparée» est permise. Elle explique cependant que seuls trois régimes soient abordés: ceux issus du PIDCP, de la CADH et de la Convention EDH. Car, si tous les articles précités empruntent un même modèle, seuls les organes de protection de ces trois textes ont eu véritablement l'occasion d'en préciser la portée ${ }^{11}$. Aussi peut-on considérer que ces mécanismes de protection et leurs décisions ont atteint un "degré de maturité suffisant ${ }^{12}$ pour qu'ils puissent être comparativement analysés, même si, on le verra, la jurisprudence de la Cour européenne des droits de l'homme ${ }^{13}$ reste davantage développée en la matière que celle de son homologue interaméricaine ${ }^{14}$ et du Comité des droits de l'homme ${ }^{15}$.

Dans sa thèse La constitutionnalisation des pouvoirs de crise, Vincent Souty relate l'existence de deux approches opposées relatives à l'état d'exception: l'une moniste et l'autre dualiste ${ }^{16}$. Les tenants de la première refusent d'instituer dans le champ juridique toute distinction entre période exceptionnelle et période normale. Aucun encadrement n'est préconisé pour essentiellement deux raisons. La première est qu'en imposant aux gouvernants le cadre constitutionnel normal en période de crise, ces derniers auront conscience de le violer pour y faire face et d'encourir une sanction potentielle. Ils ne l'enfreindront, de fait, qu'avec une extrême prudence. Le but visé est donc d' «éviter l'usage arbitraire de pouvoirs de crise» en posant «une présomption de non-légitimité,

3. Il vise en effet «les projets de réformes constitutionnelle et pénale en cours», notamment les «dispositions concernant l’usage des armes à feu par les forces de l'ordre», les «restrictions à la liberté d'aller et venir» ainsi que «les discussions qui se tiennent au niveau national autour de l'élargissement des cas de déchéance de la nationalité française» (T. Jagland, lettre au président de la République française).

4. Ci-après «Convention EDH», «Convention européenne» ou «Convention de Rome».

5. Cette «temporalité» ressort de tout régime international prévoyant des dérogations aux droits en cas de circonstances exceptionnelles: «les mesures adoptées doivent viser à contenir, puis à supprimer cette menace exceptionnelle. Elles ne peuvent donc qu'être provisoires et doivent disparaître en même temps que la crise est jugulée: elles doivent devenir caduques une fois l'état d'exception abrogé» (V. Souty, «Les dérogations en cas de circonstances exceptionnelles: un régime en demi-teinte», Revue trimestrielle des droits de l'homme, n 109, 2017, p. 96).

6. Le contrôle international vient compléter le contrôle opéré par les juridictions internes tout en l'encadrant. Voir nos considérations infra.

7. On peut d'ailleurs à ce titre affirmer avec Alain Pellet que les particularités attachées aux traités protégeant les droits de l'homme tiennent «moins à [leur] objet [...] qu'à l'existence d'organes de contrôle, plus répandue que dans d'autres domaines» (A. Pellet, " "Droits-de-l'hommisme" et droit international», Droits fondamentaux, $\mathrm{n}^{\circ}$ 1, juillet-décembre 2001, p. 158).

8. La Déclaration universelle des droits de l'homme, le Pacte international relatif aux droits économiques, sociaux et culturels ou encore la Charte africaine des droits de l'homme et des peuples ne contiennent pas, quant à elles, de clause dérogatoire et sont, de fait, exclus du champ de cette étude.

9. Ci-après «PIDCP» ou «Pacte».

10. Ci-après « $\mathrm{CADH} »$, «Convention américaine» ou «Pacte de San José ».

11. S'agissant de la Charte sociale européenne, il faut rappeler que le Comité européen des droits sociaux n'a pas la possibilité d'examiner des requêtes individuelles. Néanmoins, un système de réclamations collectives a été mis en place, permettant à certaines organisations non gouvernementales d'y avoir accès. Cependant, une seule réclamation collective (Comité européen des droits sociaux, recevabilité, Confédération générale grecque du travail (GSEE) c. Grèce, réclamation n $\mathrm{n}^{\mathrm{0}}$ 111/2014, 19 mai 2015) est, à notre connaissance, relative à l'article 30 de la Charte de Turin, équivalent de l'article F de la Charte révisée. S'agissant de la Charte arabe, elle institue certes un Comité arabe des droits de l'homme mais qui n'est compétent que dans le cadre d'un mécanisme de contrôle sur rapports étatiques.

12. Cette expression est empruntée à Jean-François Flauss, «La présence de la jurisprudence de la Cour suprême des États-Unis d’Amérique dans le contentieux européen des droits de l'homme», Revue trimestrielle des droits de l'homme, n 62, 2005, p. 325 .

13. Ci-après «Cour européenne» ou «Cour EDH».

14. Ci-après «Cour interaméricaine» ou «Cour IDH».

15. Si tant est que l'on puisse parler de «jurisprudence» à l'égard des «constatations» et des «observations finales» adoptées par ce dernier, qui, s'il «est certainement chargé d'une fonction juridictionnelle», ne revêt pas la qualification de juridiction dans la mesure où ses décisions «n'ont pas l'autorité de la chose jugée» (O. de Frouville, "Introduction de la quatrième partie», in Le Pacte international relatif aux droits civils et politiques. Commentaire article par article, E. Decaux (dir.), Paris, Economica, 2011, p. 628-629). Par facilité de langage, néanmoins, les termes de «juge» et de "jurisprudence» entendus lato sensu seront parfois employés à l'égard du Comité des droits de l'homme (ci-après «Comité»).

16. V. Souty, La constitutionnalisation des pouvoirs de crise. Essai de droit comparé, thèse de doctorat en droit public, université Paris 3 - Sorbonne Nouvelle, 2015, p. 52-72. 
réfragable, de la nécessité $\left[\mathrm{d}\right.$ 'y] recourir ${ }^{17}$. La deuxième est davantage pragmatique. Formant le constat que le droit est inapte à encadrer l'exception, plusieurs auteurs considèrent que cette question soit ressort du domaine politique ${ }^{18}$, soit constitue un droit naturel de l'État ${ }^{19}$, soit, enfin, est inhérente à tout texte constitutionnel, pouvant par essence être interprété de façon à sauvegarder l'existence de l'État ${ }^{20}$. Si la sauvegarde de l'ordre public impose que des mesures exceptionnelles soient adoptées, l'ordre juridique fera soit l'objet d'une suspension, soit s'autorégulera, s'accommodera de violations ponctuelles du droit qui seront - ou non - par la suite sanctionnées en fonction de leur légitimité.

À l'inverse, les tenants de l'approche dualiste font une distinction nette entre normalité et exceptionnalité. Ils plaident en faveur d'un encadrement des pouvoirs de crise en vue de renforcer l'État de droit. Les prévoir permet de sauvegarder plusieurs de ses manifestations principales:

[...] la sécurité juridique (connaissance des normes applicables par leurs destinataires), la limitation du pouvoir (hétérolimitation de l'exercice du pouvoir) et la garantie des droits et libertés ${ }^{21}$.

De ce point de vue, on peut affirmer que les régimes dérogatoires en droit international des droits de l'homme sont plutôt fondés sur ce modèle dualiste. Le but recherché par les rédacteurs des trois textes abordés est de sauvegarder le système conventionnel en encadrant le pouvoir de l'État en cas de circonstances exceptionnelles (I). Nous constaterons cependant que leur efficacité repose sur les épaules du juge, ultime garde-fou de ce système. Or, l'étude de la pratique des deux cours régionales de protection des droits de l'homme et du Comité démontre que l'étendue de leur contrôle varie (II). L'objectif d'encadrement de l'État poursuivi par les rédacteurs de ces textes risque donc d'être mis en échec et est, au moins, remis en cause par une certaine pratique.

\section{La création de clauses dérogatoires ou la consécration de l'approche dualiste des pouvoirs de crise}

La question de l'inclusion d'une clause prévoyant des dérogations s'est tout d'abord posée dans le cadre des travaux préparatoires du PIDCP, qui consacre le modèle dualiste de l'état d'exception (A). Si ce dernier sera repris par les deux conventions régionales, ce n'est cependant pas sur le même fondement (B).

\section{A. La consécration du modèle dualiste par le Pacte international relatif aux droits civils et politiques}

Contrairement aux traités internationaux précités, la Déclaration universelle des droits de l'homme ${ }^{22}$ ne contient pas de disposition relative aux «dérogations ${ }^{23}$. La question de l'adoption d'une telle clause s'est cependant posée lors de sa rédaction, suite à la transmission, le 12 juin 1947, d'un projet de Déclaration internationale des droits de l'homme au secrétaire général des Nations unies par Lord Dukeston, représentant britannique à la Commission des droits de l'homme, qui y était favorable $^{24}$. Ce dernier ayant été élu président du groupe de travail chargé de rédiger un projet de Pacte des droits de l'homme ${ }^{25}$, il n'est guère étonnant qu'on en trouve la mention dans la première version de ce qui devait devenir le PIDCP. La clause était cependant rédigée de manière très permissive puisqu'elle envisageait la suspension des droits dans leur ensemble ${ }^{26}$. Cette version initiale est à replacer dans son contexte. Cette proposition britannique était davantage motivée par des raisons pratiques et politiques que théoriques: l'idée était que le Royaume-Uni puisse se libérer de ses obligations dans sa gestion de l'empire colonial $^{27}$. Elle a néanmoins été rejetée quelques mois

17. Ibid., p. 55 .

18. Ibid., p. 56-6o.

19. Ibid., p. 6o-62.

20. Ibid., p. 62-67.

21. Ibid., p. 69.

22. Ci-après «DUDH».

23. La DUDH ne contient qu'une clause générale de «limitation» aux droits. Précisons ici que ce terme est celui employé par son article 29, $\$ 2$, qui prévoit que, «[d]ans l'exercice de ses droits et dans la jouissance de ses libertés, chacun n'est soumis qu'aux limitations établies par la loi exclusivement en vue d'assurer la reconnaissance et le respect des droits et libertés d'autrui et afin de satisfaire aux justes exigences de la morale, de l'ordre public et du bien-être général dans une société démocratique». Le PIDCP, la CADH et la Convention EDH utilisent quant à eux le terme de «restrictions", que nous reprendrons dorénavant. À ce titre, certaines ingérences étatiques sont considérées comme compatibles avec ces conventions dans la mesure où elles sont prévues par la loi, poursuivent certains buts strictement énumérés (défense de l'ordre, protection de la santé et de la morale, protection des droits et libertés d'autrui, etc.), nécessaires dans une société démocratique, et proportionnées. Sur cette distinction entre dérogation et restriction, voir M. Delmas-Marty, Raisonner la raison d'État: vers une Europe des droits de l'homme, Paris, PUF, 1989.

24. UN Doc. E/CN.4/AC.1/4, 12 juin 1947 , p. 8

25. UN Doc. E/60o, 2-17 décembre 1947, p. 8

26. Selon l'article $4, \S 1$, de ce projet: «En temps de guerre ou en cas d'autre danger public, un État peut prendre des mesures dérogeant aux obligations prévues à l'article 2 ci-dessus, dans la stricte mesure des exigences de la situation» (UN Doc. E/60o, p. 25). Or, l'article 2 auquel il est fait référence institue, entre autres, l'obligation pour les États de «veiller à ce que: (a) leurs lois garantissent à toutes les personnes relevant de leur souveraineté, qu'il s'agisse de ressortissants, d'étrangers ou d'apatrides, la jouissance de ces droits de l'Homme et de ces libertés fondamentales» (ibid.).

27. J. Reynolds, "The Long Shadow of Colonialism: The Origins of the Doctrine of Emergency in International Human Rights Law», Comparative Research in Law \& Political Economy, Research Paper no 19, Toronto, Osgoode Hall Law School, 2010, p. 21-23. Selon l'auteur, en effet, «Britain entered the drafting process for the relevant legally binding human rights instruments wary of the need to retain the necessary latitude to deal with the "emergencies" it was facing in certain parts of the empire" (p. 22-23). 
plus tard et fera, par la suite, l'objet de multiples débats et propositions d'amendements ${ }^{28}$.

Deux écoles opposées s'affrontent alors. La première, que l'on retrouve essentiellement dans les propos des représentants britanniques, cherche à restreindre au maximum les obligations conventionnelles et à permettre aux États de s'en affranchir en cas de circonstances exceptionnelles. La seconde plaide pour la suppression pure et simple de toute clause dérogatoire. Les représentants des États-Unis d'Amérique avancent, pour justifier cette position, le risque d'anéantissement des clauses de restriction aux droits ${ }^{29}$. D'autres, comme ceux du Chili, soulignent le risque de dérive autoritaire dans la mesure où les situations dans lesquelles elle pourrait être enclenchée ne sauraient être suffisamment définies ${ }^{30}$. Faute d'être en mesure d'encadrer correctement les pouvoirs de crise, il faut imposer aux États de rester soumis au Pacte en toutes circonstances. En outre, les dispositions relatives aux restrictions seraient rédigées de manière suffisamment large pour «couvrir tous les cas qui pourraient survenir en cas de guerre ou de calamité mentionnées dans cet article ${ }^{31}$. L'approche moniste est ici préconisée.

C'est finalement un compromis entre ces deux écoles, à l'origine duquel on retrouve une proposition de la France $^{32}$, qui l'emportera. Il passe par la reconnaissance d'une clause de dérogation, mais qui contient une liste d'articles ou de principes auxquels aucune dérogation ne saurait être admise. Sorte d' "exception à l'exception " ${ }^{33}$, elle permet de contenter les tenants des deux approches: les États ne peuvent se soustraire que partiellement à leurs obligations conventionnelles (seuls les droits dérogeables sont concernés).

Cette solution retenue ne sera pas sans incidence sur la rédaction des deux autres textes abordés.

\section{B. La reprise du modèle international dans les conventions régionales}

La Convention européenne est le premier texte international contraignant adopté en vue de protéger les droits de l'homme. Sa rédaction n'a par conséquent pas pu être directement influencée par le PIDCP, qui lui est postérieur, et l'a été davantage par la DUDH ${ }^{34}$. C'est pourquoi sa toute première version ne prévoyait aucune clause de dérogation et contenait une clause générale de restrictions «faisant écho à l'article 29(2) de la Déclaration universelle des droits de l'homme ${ }^{35}$. En outre, les discussions ont dans un premier temps tourné autour de la clause de l'abus de droit ${ }^{36}$. Celles relatives à l'insertion au sein du PIDCP d'une clause dérogatoire seront néanmoins rappelées par les représentants britanniques avec une acuité particulière ${ }^{37}$. La création d'une telle clause fondée sur ce modèle a alors fait l'objet de débats ${ }^{38}$, contrairement à son contenu une fois le principe acté ${ }^{39}$.

C'est peut-être là une des raisons pour lesquelles la formulation de l'article 15 de la Convention EDH est restrictive, surtout lorsqu'on la compare à celle de l'article 4 du PIDCP. Le rapport du Comité d'experts en matière de droits de l'homme chargé en octobre 1967 par le Comité des ministres du Conseil de l'Europe d'étudier la question de l'articulation entre les deux textes le souligne d'ailleurs $^{40}$. Le premier paragraphe de l'article 15 contient à ce titre plusieurs lacunes, certaines plus importantes que d'autres ${ }^{41}$, parmi lesquelles on trouve l'absence de deux mentions: l'obligation de proclamer «par un acte officiel» le danger public en cause et l'exigence de nondiscrimination ${ }^{42}$. Le paragraphe 2 de l'article 15 énumère ensuite beaucoup moins de droits indérogeables puisque «l'interdiction de l'emprisonnement pour dettes civiles», le «droit à la reconnaissance de la personnalité juridique»

28. J. Reynolds, "The Long Shadow of Colonialism... », p. 27-29.

29. Sur ce terme de «restriction», voir supra, note 23 .

30. UN Doc. E/CN.4/SR.195, 29 mai 1950, p. 13.

31. Propos du représentant chilien. L'auteure précise ici que, sauf mention contraire, les traductions sont les siennes.

32. J. Reynolds, "The Long Shadow of Colonialism...», p. 28.

33. V. Souty, La constitutionnalisation des pouvoirs de crise..., p. 108.

34. M. Rota, L'interprétation des Conventions américaine et européenne des droits de l'homme. Analyse comparée de la jurisprudence des deux Cours de protection des droits de l'homme, Issy-les-Moulineaux, LGDJ, 2018, p. 31-40.

35. J. Reynolds, "The Long Shadow of Colonialism... », p. 23. Voir nos considérations supra, note 23.

36. Le risque de dérive autoritaire devait être prévenu par le biais de cette clause qu'on a souhaité étendre aux États pour éviter que la Convention ne puisse être utilisée au détriment des droits et libertés. Voir V. Souty, La constitutionnalisation des pouvoirs de crise..., p. 105-107.

37. J. Reynolds, «The Long Shadow of Colonialism...», p. 27.

38. Ibid., p. 24. Pour une analyse détaillée des travaux préparatoires de cette disposition, ibid., p. 23-26.

39. Ibid., p. 28 .

40. Comité d'experts en matière de droits de l'homme, Problèmes découlant de la coexistence des pactes des Nations unies relatifs aux droits de l'homme et de la Convention européenne des droits de l'homme, 25 septembre 1970, doc. H (70) 7, en ligne: http://echr.coe.int/library/digdoc/ DG2/HDOCS/COE-1970-FR-H\%2870\%297.pdf. Ce document est en outre cité dans le rapport explicatif du Protocole n 7 à la Convention EDH, adopté suite à la recommandation faite par l'Assemblée parlementaire «de s'efforcer d'insérer le plus grand nombre possible de dispositions positives du Pacte des Nations unies dans la Convention européenne des Droits de l'Homme» (article 12-c-ii de la recommandation 791 (1976) relative à la protection des droits de l'homme en Europe, adoptée par l'Assemblée parlementaire du Conseil de l'Europe le 17 septembre 1976).

41. Le Comité d'experts souligne que si l'article $4, \$ 1$ du Pacte ne permet pas de dérogations "en cas de guerre", ce que fait l'article 15 de la Convention EDH, cette hypothèse peut être incluse dans l'expression consacrée: «danger public exceptionnel [qui] menace l'existence de la nation" (recommandation 791 (1976), p. 18). De même, la différence existante entre les deux versions françaises de ces textes (danger public exceptionnel ou non et menaçant «la vie de la nation» ou la nation elle-même) disparaissant en anglais, elle ne semble pas non plus décisive (ibid., p. 19).

42. Recommandation 791 (1976) 
et «la liberté de pensée, de conscience et de religion " ${ }^{43}$ ne sont pas visés. Il prévoit en outre que le droit à la vie puisse faire l'objet de dérogations en cas de «décès résultant d'actes licites de guerre ${ }^{44}$. Enfin, le dernier paragraphe ne prévoit pas d'obligation de signaler «aussitôt » l'adoption de dérogations sur son fondement. Le texte conventionnel européen est donc moins protecteur.

Il en va en revanche différemment s'agissant de la Convention américaine, dont les rédacteurs ont été directement influencés par le Pacte. Car, si les origines de cette Convention sont à rechercher au sein d'un panaméricanisme qui remonte bien avant la Seconde Guerre mondiale ${ }^{45}$, elle a aussi hérité des différentes avancées en matière de protection des droits au plan international. Il faut ici rappeler que le projet d'élaboration d'une convention et d'une cour proprement régionales remonte à 1945 , mais que la situation de crise qu'a connue l'Organisation des États américains (OEA) a ralenti ce processus. Son Conseil ne sera alors ressaisi de la question de l'élaboration d'une convention qu'en 1959 et le projet est relancé lors de la $\mathrm{V}^{\mathrm{e}}$ réunion des ministres des Affaires étrangères du mois d'août de la même année ${ }^{46}$. Or, c'est à cette période qu'est discutée l'adoption des deux pactes onusiens. C'est pourquoi il a été choisi de conditionner la création d'une convention régionale à sa compatibilité et à sa complémentarité avec ces derniers ${ }^{47}$. Sa rédaction est alors adaptée en vue de la mettre en adéquation avec les pactes ${ }^{48}$ et on retrouve par conséquent une clause dérogatoire dans son texte dès l'origine, présentant beaucoup moins de différences rédactionnelles.

Néanmoins, le contexte dictatorial auquel ont eu à faire face la plupart des États d'Amérique du Sud ${ }^{49}$ a amené ses rédacteurs à être beaucoup plus précis s'agissant de la liste des droits indérogeables. Il faut avoir à l'esprit que la théorie des circonstances exceptionnelles a été, dans la plupart de ces États, déviée en vue de justifier la mise en place de régimes autoritaires ${ }^{50}$. Le but des rédacteurs du
Pacte de San José a donc été d'encadrer davantage les États, en prévoyant davantage de droits indérogeables. En plus des droits à la reconnaissance de la personnalité juridique, à la vie, à l'intégrité de la personne, de l'interdiction de l'esclavage et de la servitude, du principe de légalité et de non-rétroactivité de la loi pénale, de la liberté de conscience et de religion - droits indérogeables qu'on retrouve aussi dans le PIDCP -, ils y ajoutent la protection de la famille, le droit à un nom, le droit de l'enfant, le droit à une nationalité et les droits politiques. L'article $27, \$ 2$ précise en outre que «les garanties indispensables à la protection des droits susvisés» sont insusceptibles de dérogations.

Notons enfin que le passé colonial des États parties à ce texte est sans doute à l'origine d'une ouverture des circonstances visées. Alors que le texte du Pacte autorise les dérogations « $[\mathrm{d}]$ ans le cas où un danger public exceptionnel menace l'existence de la nation", la CADH se réfère aussi à d'autres «situations de crise» et, au lieu de viser uniquement «la vie de la nation», il envisage aussi la menace à «l'indépendance et à la sécurité de l'État».

Malgré ces différences, on peut affirmer que les trois conventions empruntent in fine un même modèle. L'approche dualiste des pouvoirs de crise est consacrée. Cependant, l'efficacité de ces clauses dérogatoires repose sur l'existence d'un contrôle international. Ce sont en effet les juges régionaux et le Comité des droits de l'homme qui vont vérifier que les conditions de mise en œuvre de ces dispositions sont bien respectées. En outre, en tant que juges subsidiaires, ils s'attacheront à vérifier que le contrôle opéré par le juge interne sur les mesures dérogatoires est suffisant ${ }^{51}$. Aussi assiste-t-on depuis l'adoption de ces dispositions à ce que Mireille Delmas-Marty a pu appeler « des mutations de l'État de droit dans un monde qui s'internationalise $»^{52}$. En effet,

[...] les dérives autoritaires sont censurées tantôt par le juge national, devenu gardien d'un droit international qui l'émancipe du droit national, tantôt par le juge

43. Ibid., p. 20

44. Ibid., p. 8

45. M. Rota, L'interprétation des Conventions..., p. 33. Rappelons à ce titre que les rédacteurs de la Convention ont bien plus été influencés par la Déclaration américaine des droits et devoirs de l'homme du 2 mai 1948 que par la DUDH. La Déclaration américaine des droits et devoirs de l'homme ne contient cependant pas de clause dérogatoire. Son article XXVIII prévoit seulement que " [l]es droits de chaque homme sont limités par les droits des autres, par la sécurité de tous et par les justes exigences du bien-être général et du développement de la démocratie».

46. Suite à cette réunion, la résolution VIII est adoptée et il est demandé au Conseil interaméricain de jurisconsultes de créer un projet de Convention américaine relative aux droits de l'homme prévoyant une cour et une commission. Le texte de cette résolution est reproduit en Annexe 2 de l'ouvrage de K. Vasak, La Commission interaméricaine des droits de l'homme. La protection internationale des droits de l'homme sur le continent américain, Paris, Librairie générale de droit et de jurisprudence, 1968, p. 214.

47. Commission interaméricaine des droits de l'homme, «Estudio comparativo entre los pactos de las naciones unidas sobre derechos civiles, políticos, económicos, sociales y culturales y los proyectos de convención interamericana sobre derechos humanos ", OEA/Ser.L./V/II.19, doc. 18, 24 juin 1968, Anuario interamericano de derechos humanos, n 58, 1968, p. 207 et 211, aussi appelé «rapport Dunshee de Abranches» du nom du président du groupe de travail de la Commission chargé d'élaborer ce rapport.

48. Ibid., p. 168-213.

49. M. Rota, L'interprétation des Conventions américaine et européenne des droits de l'homme. Analyse comparée de la jurisprudence des deux Cours de protection des droits de l'homme, thèse de doctorat en droit public, université de Caen Normandie, 2013, Annexe 2, p. $683-684$.

50. C'est pour faire face à la menace communiste que la prise de pouvoir par les militaires a été autorisée. Voir V. Souty, La constitutionnalisation des pouvoirs de crise..., p. 47-49.

51. S'agissant de la question de l'articulation entre le contrôle opéré par la Cour européenne des droits de l'homme et le juge national, voir P. Wachsmann, "Contrôle des mesures prises au titre de l'état d'urgence et Convention européenne des droits de l'homme», L'actualité juridique. Droit administratif, $\mathrm{n}^{\circ}$ 43, 2016, p. 2425-2433.

52. M. Delmas-Marty, «Quand l'Europe raisonne la raison d'État», Revue Projet, nº 324-325, 2011, p. 21-22, en ligne: http://www.cairn.info/revueprojet-2011-5-page-16.htm. 
international lui-même. Dans l'un et l'autre cas, l'État de droit n'est plus identifié par référence au seul droit étatique, mais à un droit interétatique, voire supra-étatique, qui enrichit mais complexifie les dispositifs de résistance à la raison d'État ${ }^{53}$.

Le rôle joué par l'organe international de contrôle est primordial. De lui dépend l'encadrement qui motive l'adoption de ces clauses. Il nous faut alors étudier sa pratique en vue de déterminer si l'objectif poursuivi par les négociateurs est atteint.

\section{Un encadrement variable en fonction de la pratique des organes de contrôle}

L'examen de la «jurisprudence» du Comité et des deux cours régionales des droits de l'homme démontre que la pratique est variable s'agissant de l'encadrement des États issu de l'interprétation de ces clauses de dérogation. À ce titre, la jurisprudence de la Cour européenne apparaît nettement en retrait par rapport à celle des deux autres organes. Ceux-ci n'hésitent pas, en effet, à étendre la liste des droits indérogeables (A); ils exercent un contrôle plus poussé sur la mise en œuvre de la clause dérogatoire (B); on ne peut, enfin, aucunement leur reprocher un retour à l'approche moniste en dehors de son déclenchement (C).

\section{A. L'extension des normes indérogeables par le Comité des droits de l'homme et la Cour interaméricaine}

Dans son Observation générale $n^{\circ} 29$, le Comité affirme que

Dans les dispositions du Pacte qui ne figurent pas au paragraphe 2 de l'article 4 , il y a des éléments qui, de l'avis du Comité, ne peuvent pas faire l'objet d'une dérogation licite en vertu de l'article $4^{54}$.

Il ne considère par conséquent pas la liste dressée par cette disposition comme limitative et mentionne, comme l' «un des critères possibles» pour déterminer la légitimité de dérogations, «la définition de certaines violations des droits de l'homme en tant que crimes contre l'humanité » ${ }^{55}$. Mais ce critère n'est pas le seul. Le Comité prend également acte de l'évolution du droit international général en la matière et des normes de jus cogens. À partir de là, il liste cinq « exemples représentatifs» d'autres droits, obligations et prohibitions ne pouvant pas non plus faire l'objet de dérogations ${ }^{56}$.

La Cour interaméricaine procède de la même façon. Elle estime qu'il ne peut être dérogé aux articles $25, \$ 1$ et $7, \$ 6$ de la Convention protégeant le droit d'accéder à un tribunal et ses garanties procédurales alors même qu'ils ne figurent pas dans le deuxième paragraphe de l'article 27. Dans la mesure où il s'agit de garanties judiciaires indispensables à la protection de droits conventionnellement qualifiés d'indérogeables, elle affirme qu'ils doivent, eux aussi, revêtir cette qualité ${ }^{57}$. Elle est arrivée à cette même conclusion plus récemment s'agissant de l'article $7, \S 3$ qui prohibe les détentions et arrestations arbitraires, en se fondant, entre autres, sur la pratique du Comité ${ }^{58}$.

Cette tendance commune aux deux organes s'explique par le fait qu'ils se positionnent en tant que véritables juges de droit international. Si cette spécificité peut aisément se comprendre s'agissant du Comité, institué pour protéger une convention élaborée dans le cadre de l'ONU, elle peut interroger s'agissant de la Cour de San José. Mais elle s'explique par la vision spécifique qu'elle a de son propre rôle et qui diffère de celle du juge européen ${ }^{59}$.

La Cour interaméricaine peut, en effet, être qualifiée de juge régional de droit universel. De fait, elle se pose en véritable guide pour les États, en vue de construire avec eux des droits internes protecteurs des libertés, en partant des avancées du droit international général. La collectivité à laquelle elle s'adresse est en outre une collectivité internationale et elle n'hésite pas, par conséquent, à indexer l'interprétation de la Convention sur le consensus émergeant en son $\operatorname{sein}^{60}$. Elle se doit donc d'intégrer toute avancée issue du droit international dont elle se veut le «relais ${ }^{61}$.

En Europe, il s'agirait plutôt d'un mouvement inverse. La Cour apparaît davantage comme juge international de droit régional. Elle s'attache à harmoniser les droits internes, et, à partir de là, bâtir un ordre proprement régional. Le consensus visé n'est donc plus le même: c'est celui qu'elle décèle au sein d'une collectivité proprement régionale ${ }^{62}$.

53. M. Delmas-Marty, "Quand l'Europe raisonne la raison d'État», p. 22.

54. Comité des droits de l’homme, Observation générale nº 29, États d'urgence (art. 4), 31 août 2001, CCPR/C/21/Rev.1/Add.11, \$13.

55. Ibid., $\$ 12$.

56. Il s'agit de l'obligation de traiter «avec humanité et avec le respect de la dignité inhérente à la personne humaine» toute personne privée de sa liberté; «l'interdiction de la prise d'otages, des enlèvements ou des détentions non reconnues»; certains aspects de la «protection internationale des droits des personnes appartenant à des minorités »; la prohibition de «la déportation ou le transfert forcé de population » et de «la propagande en faveur de la guerre ou [...] des appels à la haine nationale, raciale ou religieuse» (ibid., $\$ 13$ ).

57. Cour IDH, El Hábeas Corpus bajo suspensión de garantías (arts. 27.2, 25.1 y 7.6 Convención Americana sobre Derechos Humanos), opinion consultative $\mathrm{n}^{\circ} \mathrm{OC}-8 / 87,30$ janvier 1987, série A, n $8, \$ 24$. Voir aussi Cour IDH, Neira Alegría et autres $c$. Pérou, fond, 19 janvier 1995, série C, $\mathrm{n}^{\circ} 20, \$ 82-84$

58. Cour IDH, Osorio Rivera et autres $c$. Pérou, exceptions préliminaires, fond, réparations et dépens, 26 novembre 2013 , série $\mathrm{C}, \mathrm{n}^{\circ} 274, \S 120$.

59. M. Rota, L'interprétation des Conventions..., Issy-les-Moulineaux, LGDJ, 2018, p. 27-229.

60. Ibid., p. 359-366

61. Ce terme est emprunté à L. Hennebel, La Convention américaine des droits de l'homme. Mécanismes de protection et étendue des droits et libertés, Bruxelles, Bruylant, 2007, p. 37

62. M. Rota, L'interprétation des Conventions..., p. 286-293. 
Il s'agit dès lors d'un mouvement ascendant et la Cour se montre beaucoup plus respectueuse de la compétence des États. Cela explique sans doute pourquoi elle ne procède pas à une telle ouverture des normes indérogeables.

Le juge européen se situe aussi en retrait par rapport au Comité et à la Cour interaméricaine, ce qui est par ailleurs confirmé au regard de l'étendue de son contrôle sur l'article 15.

\section{B. L'exercice d'un contrôle plus poussé par le Comité et la Cour interaméricaine sur la mise en œuvre de la clause dérogatoire}

La principale critique adressée à la jurisprudence de la Cour européenne est le caractère trop limité de son contrôle sur l'article $15^{63}$ du fait de la large marge d'appréciation qu'elle laisse aux États en la matière. C'est d'ailleurs à l'occasion d'une affaire relative à cette disposition ${ }^{64}$ que l'ancienne Commission européenne des droits de l'homme a créé la «notion toute prétorienne de marge d'appréciation » ${ }^{65}$, manifestation la plus directe de son caractère subsidiaire mais signe aussi de son autolimitation ${ }^{66}$. Or, la Cour concède aux États une telle marge, qui plus est étendue, tant dans le cadre de la qualification des circonstances que dans celui de l'étendue des dérogations adoptées. Selon elle en effet,

Il incombe d'abord à chaque État contractant, responsable de «la vie de (sa) nation», de déterminer si un «danger public» la menace et, dans l'affirmative, jusqu'où il faut aller pour essayer de le dissiper ${ }^{67}$.

Elle poursuit en affirmant que, dans la mesure où les autorités nationales sont «en contact direct et constant avec les réalités pressantes du moment», elles
[...] se trouvent en principe mieux placées que le juge international pour se prononcer sur la présence de pareil danger comme sur la nature et l'étendue de dérogations nécessaires pour le conjurer ${ }^{68}$.

C'est pourquoi elles peuvent bénéficier d'une telle marge, qui va cependant "de pair avec un contrôle européen », pour reprendre l'expression consacrée par la suite ${ }^{69}$. Les États «ne jouissent pas pour autant d'un pouvoir illimité en ce domaine $»^{70}$ et la Cour va donc pallier tout risque d'arbitraire dans la qualification des circonstances. Elle le fait aussi en vérifiant que les mesures adoptées n'ont pas «excédé la "stricte mesure" des exigences de la crise »" condition fixée par l'article 15. Le juge européen conserve alors le pouvoir d'apprécier la stricte proportionnalité des mesures adoptées par rapport au but poursuivi. Aussi, l'État devra

[...] démontrer le caractère indispensable, inévitable de l'atteinte, ce qui signifie notamment que le danger ne peut être écarté par d'autres moyens moins attentatoires aux droits et libertés ${ }^{72}$.

Ce contrôle de proportionnalité a cependant pu faire l'objet de critiques. Dans l'affaire A. c. Royaume-Uni, par exemple, la Cour refuse de considérer que les mesures dérogatoires doivent avoir un caractère « exceptionnel et provisoire ${ }^{73}$. Si la menace est persistante, elles ne sauraient être sanctionnées pour ce seul motif. Or, la Cour va ici clairement à l'encontre de la pratique du Comité des droits de l'homme, qu'elle cite pourtant ${ }^{74}$.

Ce dernier, tout comme la Cour interaméricaine, est en effet plus protecteur. S'agissant de cette dernière, elle affirme certes que les autorités nationales doivent au premier chef évaluer si la situation exige le déclenchement de l'état d'exception dans l'ordre interne et la conformité des mesures prises au regard de la Convention ${ }^{75}$. Elle fait cependant fi de toute référence à l'existence d'une

63. Pour une analyse détaillée du contrôle opéré par la Cour, voir A. Petropoulou, Liberté et sécurité: les mesures antiterroristes et la Cour européenne des droits de l'homme, Paris, A. Pedone, 2014.

64. Commission EDH, 26 septembre 1958, Grèce c. Royaume-Uni, nº 176/56.

65. F. Sudre, Droit européen et international des droits de l'homme, Paris, PUF, 2012, p. 231. Pour une critique de ce caractère prétorien, se reporter à J. García Roca, «La muy discrecional doctrina del margen de apreciación nacional según el Tribunal Europeo de Derechos Humanos », Teoría y realidad constitucional, $\mathrm{n}^{\circ} 20,2007, \mathrm{p} \cdot 117-143$

66. Comme le constatent les professeures Mireille Delmas-Marty et Marie-Laure Izorche, cette «marge concédée par le juge lui-même [est] une sorte d'autolimitation de ses propres pouvoirs normatifs» (M. Delmas-Marty, M.-L. Izorche, "Marge nationale d'appréciation et internationalisation du droit. Réflexions sur la validité formelle d’un droit commun pluraliste», Revue internationale de droit comparé, vol. 52, n 4 , 2000, p. 754). C'est là sans doute pourquoi la Cour interaméricaine refuse de la consacrer de manière explicite. Notons toutefois que, même si l'expression n'est volontairement pas employée, la Cour consacre tout de même, selon nous, une telle marge aux États dans certains domaines (M. Rota, L'interprétation des Conventions..., p. 217-226).

67. Cour EDH, 18 janvier 1978, Irlande c. Royaume-Uni, n 5310/71, \$207

68. Ibid.

69. Cour EDH, 7 décembre 1976, Handyside c. Royaume-Uni, nº 5493/72, \$ 49.

70. Cour EDH, 18 janvier 1978, Irlande c. Royaume-Uni, $\$ 207$

71. Ibid.

72. F. Tulkens, «Dérogations», in Dictionnaire des droits de l’homme, J. Andriantsimbazovina, H. Gaudin, J.-P. Marguénaud, S. Rials, F. Sudre (dir.), Paris, PUF, 2008, p. 269. Dans ce cadre, le juge doit «attacher le poids qui convient à des facteurs pertinents tels que la nature des droits touchés par la dérogation, la durée de l'état d'urgence et les circonstances qui l'ont créé» (Cour EDH, 2 ${ }^{\mathrm{e}}$ section, 17 décembre 2002, A. c. Royaume-Uni, $\left.\mathrm{n}^{\circ} 35373 / 97, \$ 173\right)$.

73. Cour EDH, $2^{\mathrm{e}}$ section, 17 décembre 2002, A. c. Royaume-Uni, $\$ 178$.

74. Ibid., $\$ 109$. Selon le Comité, en effet, «[l] es mesures dérogeant aux dispositions du pacte doivent avoir un caractère exceptionnel et provisoire» (Comité des droits de l'homme, Observation générale nº $29, \$ 2$ ).

75. Cour IDH, J. c. Pérou, exceptions préliminaires, fond, réparations et dépens, 27 novembre 2013, série C, $\mathrm{n}^{\circ} 275, \$ 137$. 
marge d'appréciation. Elle opère alors un contrôle entier sur les deux aspects - qualification des circonstances et proportionnalité des mesures adoptées - dont l'examen est d'ailleurs entremêlé ${ }^{6}$. Les juges de San José s'alignent donc sur la pratique du Comité, qui semble, lui aussi, préconiser un tel contrôle ${ }^{77}$.

Cette jurisprudence peut s'expliquer par le passé dictatorial des États sud-américains. Il faut en effet avoir en tête que la prise de pouvoir par les militaires a été «systématiquement justifiée par la nécessité de faire face à une menace pour l'État ${ }^{78}$. Le principal but poursuivi par la Cour est donc d'éviter que les États ne se saisissent de ces clauses pour en abuser. Elle affirme aussi qu'elle

[...] ne peut faire abstraction des abus auxquels peuvent donner lieu et auxquels, de fait, ont donné lieu dans notre hémisphère, l'application de mesures d'exception lorsqu'elles ne sont pas strictement justifiées au regard des critères qui orientent l'article $27^{79}$.

Ce mécanisme ne saurait en outre ni comporter «la suspension temporaire de l'État de droit» ni «autoriser les gouvernants à agir en marge de la légalité, à laquelle ils doivent à tout moment se soumettre ${ }^{80}$.

Or, lorsqu'on s'attarde sur la motivation de son homologue européen, on constate que la nécessaire sauvegarde de l'ordre public - face aux «menaces» que subirait «la vie de la nation » et dont les États sont les garants - ressort comme préoccupation première. La différence de contrôle qui en découle implique une certaine rétrocession d'un pouvoir à l'État dont il pourra par conséquent potentiellement abuser et ce, légalement, puisque fondé sur l'article 15 de la Convention. Poussant cette logique jusqu'à son terme, le caractère conventionnel de la dérogation pourrait même devenir un élément de légitimation de plus, le juge devenant ainsi le complice d'une telle déviance. Sans pouvoir tout de même aller jusque-là s'agissant de la jurisprudence européenne, on est à même de saisir les risques que peut présenter un contrôle trop timoré.
L'objectif initial d'encadrement des pouvoirs de l'État porté par l'approche dualiste des pouvoirs de crise est mis à mal. Si cet encadrement existe, il n'est que minimal et tranche avec celui préconisé par la Cour interaméricaine. Il est même totalement détourné dans d'autres affaires dans lesquelles les États adoptent des mesures exceptionnelles mais sans que l'article 15 ne soit enclenché.

\section{Le retour à l'approche moniste en dehors du déclenchement de la clause dérogatoire: une tendance propre au juge européen}

\section{Pour Vincent Souty, l'}

[...] intérêt du mécanisme institué par les clauses de dérogation ne peut être assuré que si celui-ci constitue le seul moyen pour l'État d'exercer des ingérences aggravées dans les droits et libertés conventionnellement garantis ${ }^{81}$.

Or, il apparaît que de telles ingérences sont possibles en dehors du déclenchement de l'article 15 de la Convention de Rome.

Pour le comprendre, il faut rappeler que ces clauses posent une obligation de notification à la collectivité des États parties. En dehors d'un tel acte, deux solutions s'offrent alors à l'organe de contrôle: soit il accepte d'examiner tout de même l'affaire sous l'angle de la clause dérogatoire en qualifiant la mesure attentatoire aux droits protégés de dérogation, soit il le refuse et la considère comme une restriction. De ce point de vue, si le Comité des droits de l'homme ${ }^{82}$ et la Cour interaméricaine optent pour la première option ${ }^{83}$, il en va différemment de la Cour européenne. A priori, celle-ci serait donc plus protectrice dans la mesure où le régime juridique attaché aux dérogations autorise des atteintes plus importantes aux droits que celui attaché aux restrictions.

Néanmoins, l'étude de la jurisprudence européenne en la matière démontre que l'existence même de circonstances exceptionnelles implique un contrôle moins approfondi

76. Cour IDH, J. c. Pérou, \$137-147.

77. Dans son Observation générale $n^{\circ}$ 29, le Comité affirme que «les États doivent agir dans le cadre de leur constitution et des dispositions législatives qui régissent l'exercice des pouvoirs exceptionnels» et qu' «il appartient au Comité de vérifier que les lois en question permettent et garantissent le respect de l'article 4 " notamment au regard des circonstances couvertes (Comité des droits de l’homme, Observation générale n $29, \$ 2$ ). Il rappelle à ce titre que: «Tout trouble ou toute catastrophe n'entre pas automatiquement dans la catégorie d'un danger public exceptionnel qui menace l'existence de la nation, selon la définition du paragraphe 1 de l'article 4 » (ibid., $\$ 3$ ). Dans de nombreuses observations finales adoptées dans le cadre de son contrôle sur rapports étatiques, il constate que le droit interne permet de déclencher l'état d'exception en dehors de ces situations et qu'il n'est donc pas conforme à l'article 4. Dans ses observations finales adressées à la Colombie en 1997, par exemple, il «se déclare inquiet de voir que les autorités recourent encore fréquemment à la déclaration d'état d'exception et, ce, rarement en conformité avec le paragraphe 1 de l'article 4 du Pacte qui prévoit que de telles déclarations ne peuvent être faites que si un danger menace la vie et l'existence de la nation" (Comité des droits de l'homme, Observations finales (Colombie), 1997, CCPR/C/79/Add.76, \$25). Notons par ailleurs que le Comité couple, lui aussi, l'examen de la qualification des circonstances avec celui de la proportionnalité des mesures prises pour y faire face. Voir, par exemple, les observations finales suivantes: Comité des droits de l'homme, Observations finales (Liban), 1997, CCPR/C/79/Add.78, \$10; Observations finales (Uruguay), 1998, CCPR/C/79/Add.90, \$ 8; Observations finales (Israël), CCPR/C/79/Add.93, \$11.

78. V. Souty, La constitutionnalisation des pouvoirs de crise..., p. 47.

79. Cour IDH, El Hábeas Corpus..., \$20.

80. Ibid., $\$ 24$; repris au contentieux: Cour IDH, J. c. Pérou, $\$ 125$.

81. V. Souty, «Les dérogations en cas de circonstances exceptionnelles...», p. 102.

82. Le Comité affirme en effet qu'il «a le devoir d'examiner le droit et la pratique d'un État partie en vue de s'assurer que l'article 4 est respecté, que l'État partie ait ou n'ait pas fait parvenir la notification" (Comité des droits de l'homme, Observation générale $n^{\circ} 29, \$ 17$ ).

83. Dans l'affaire J. c. Pérou, bien que la Cour constate que l'État n’a pas procédé à une telle notification (Cour IDH, J. c. Pérou, $\$ 124)$, elle accepte de qualifier les mesures en cause de dérogations au regard de l'enclenchement, au niveau interne, des pouvoirs de crise ( $\$ 137-147)$. 
sur ces restrictions - qui ne seraient donc pas des restrictions ordinaires. Dans la mesure où elles ont pour objet de répondre à ces circonstances, ces dernières ne peuvent être ignorées, ni placées au second plan ${ }^{84}$. Les juges de Strasbourg acceptent de prendre en considération le «cadre général de l'affaire ${ }^{85}$ lorsqu'ils opèrent leur test de compatibilité conventionnelle et d'être, par conséquent, plus indulgents vis-à-vis des États ${ }^{86}$. Si cette critique ${ }^{87}$ nous paraît légitime, précisons toutefois que le contrôle du juge reste plus poussé que dans le cadre d'une dérogation, qui n'est pas soumise à l'examen des critères de légalité et de nécessité dans une société démocratique notamment. Cette interprétation de la Cour permet certes aux États de commettre des «ingérences aggravées », pour reprendre l'expression de Vincent Souty ${ }^{88}$, mais pas à la hauteur des dérogations.

L'auteur estime que la Cour européenne va encore plus loin dans sa jurisprudence Hassan c. Royaume-Uni dans laquelle, malgré l'absence de notification, elle «va néanmoins appliquer un mécanisme dérogatoire en matière de privation de liberté ${ }^{89}$ dans un contexte de conflit international ${ }^{90}$. Il ne nous semble cependant pas que la Cour accepte de le faire. Elle consacre en revanche une nouvelle exception au principe selon lequel «Nul ne peut être privé de sa liberté ${ }^{91}$, qu'elle ajoute au texte de la Convention en se fondant sur une interprétation de l'article 5 au regard « du contexte et des règles du droit international humanitaire $»^{92}$. Si cette « réécriture prétorienne d'une disposition expresse du texte conventionnel» peut faire l'objet de critiques puisque moins protectrice ${ }^{93}$, elle ne saurait être interprétée comme consacrant l'existence - même implicite - d'une dérogation ${ }^{94}$. Le contrôle du juge sur une exception est par ailleurs plus complet. Véritable contrôle de conformité ${ }^{95}$, il porte aussi sur le respect des garanties figurant aux $\S_{3}$ à 5 de l'article 5 . Dans le cadre d'une dérogation, qui, par essence, s'applique à cette disposition dans son ensemble, il n'est pas certain que le juge admette de se livrer à un tel contrôle et pour cause: les garanties offertes par ces paragraphes ne bénéficient pas d'un caractère indérogeable.

L'intérêt de ces différences de qualification nous paraît alors moins résider dans l'intensité du contrôle du juge international que dans l'institutionnalisation au sein même de la Convention de restrictions aggravées ou d'exceptions qui sont permanentes. À partir du moment où l'exception est conforme aux conditions énoncées par le texte (s'agissant de l'article 5 , que la détention respecte les $\$ 3$ à 5) et que la restriction est considérée comme compatible avec la Convention puisque prévue par la loi, poursuivant un but légitime, nécessaire dans une société démocratique et proportionnée, elles peuvent demeurer dans l'ordre juridique de l'État quelles que soient les circonstances. Si les dérogations sont potentiellement plus sévères, elles ont le mérite d'être temporaires. Ce retour à l'approche moniste ${ }^{96}$ provoque donc « une contamination progressive de la normalité par l'exception ${ }^{97}$ à notre sens critiquable.

Au regard de ces déviances possibles, l'analyse de la pratique de ces trois organes interroge sur la possibilité réelle pour le droit d'encadrer l'exceptionnel. La jurisprudence européenne atteste des limites que peut présenter un tel encadrement. Mais son caractère moins protecteur vient aussi poser la question de la légitimité d'un juge international pour opérer un tel contrôle. Le juge interaméricain, en bénéficiant d'une légitimité plus accrue face aux États ${ }^{98}$, n'hésite pas à se poser comme véritable rempart contre leurs déviances potentielles. Il en va plus difficilement s'agissant du Comité, qui, même s'il est tout aussi protecteur, et en raison de l'absence de pouvoir de sanction, fait l'objet d'une « indifférence [...] particulièrement regrettable aussi bien sur le plan pratique que théorique ${ }^{99}$. «Raisonner la raison d'État », pour reprendre l'expression de Mireille Delmas-Marty ${ }^{100}$, ne nous semble donc envisageable que si les États sont disposés à entendre raison.

84. Même s'il le critique, Vincent Souty reconnaît qu'«il apparaît difficilement réaliste de demander au juge de trancher un litige sans tenir compte du contexte dans lequel il se déroule, d'autant plus lorsqu'un tel litige s'inscrit dans le cadre d'une situation de crise» (V. Souty, «Les dérogations en cas de circonstances exceptionnelles...", p. 102).

85. Cour EDH, 29 novembre 1988, Brogan et autres c. Royaume-Uni, no 11209/84, 11234/84, 11266/84 et 11386/85, $\$ 48$.

86. Voir sur ce point l'analyse de V. Souty, La constitutionnalisation des pouvoirs de crise..., p. 115-120.

87. Ibid. Voir aussi, du même auteur, "Les dérogations en cas de circonstances exceptionnelles... », p. 102-105.

88. V. Souty, «Les dérogations en cas de circonstances exceptionnelles...», p. 102.

89. Ibid., p. 105

90. L'affaire concerne la détention d'un ressortissant irakien, capturé par des soldats britanniques pendant l'invasion de l'Irak, le 23 avril 2003.

91. L'article $5, \$ 1$ prévoit en effet six exceptions à ce principe tandis que les $\$ 3$ à 5 prévoient une liste de garanties pour éviter toute arrestation ou détention arbitraire.

92. Cour EDH, GC, 16 septembre 2014, Hassan c. Royaume-Uni, nº 29750/o9, \$103.

93. N. Hervieu, «La jurisprudence européenne sur les opérations militaires à l'épreuve du feu », La revue des droits de l'homme, 20 octobre 2014 en ligne: http://revdh.revues.org/89o.

94. La Cour développe d'ailleurs bien cet aspect qui se justifie au regard de la «pratique des Hautes Parties contractantes [qui] est de ne pas notifier de dérogation à leurs obligations découlant de l'article 5 lorsqu'elles incarcèrent des personnes sur la base des troisième et quatrième Conventions de Genève en période de conflit armé international» (Cour EDH, GC, 16 septembre 2014, Hassan c. Royaume-Uni, \$101).

95. À l'inverse, les restrictions et dérogations font l'objet d'une compatibilité seulement. Sur ces différences de qualifications et les conséquences qu'elles emportent voir l'ouvrage de M. Delmas-Marty, Raisonner la raison d'État...

96. La Cour applique le régime dédié aux situations normales à des affaires au sein desquelles, de fait, les États ont activé des pouvoirs de crise pour faire face à des circonstances exceptionnelles.

97. V. Souty, "Les dérogations en cas de circonstances exceptionnelles... », p. 106.

98. M. Rota, L'interprétation des conventions..., p. 52-82.

99. E. Decaux, "Avant-propos», in Le Pacte international..

100. M. Delmas-Marty, Raisonner la raison d'État... 